\title{
Modelo de supervivencia para analizar el abandono escolar de la URACCAN-Nueva Guinea
}

\author{
Survival model to analyze school dropout from URACCAN, Nueva Guinea Campus
}

Moisés Medina López ${ }^{1}$

Tonys Romero Díaz ${ }^{2}$

\section{Resumen}

El abandono escolar afecta a todas las universidades en el mundo entero. En esta investigación se explican las características que poseen los estudiantes que abandonan las carreras de la universidad URACCAN-Nueva Guinea, corte 2013-2017. Además, se propone un modelo de retención estudiantil para esta universidad. Esta investigación se realizó con dos muestras. El primer objetivo se desarrolló con todos los 347 estudiantes que se matricularon en el primer año de su carrera en el 2013. El segundo y tercer objetivo, se trabajó con 137 estudiantes retirados. Se usó el método mixto y un alcance correlacional, fenomenológico y por su ubicación temporal es un estudio transversal. En este estudio se encontró que las variables que tienen influencia estadística sobre el abandono estudiantil son la asignatura que el estudiante escoge, el número de asignaturas reprobadas y el promedio obtenido en la URACCAN. A su vez las variables estudiadas y que no influyen estadísticamente en el retiro son: género, estado civil, zona de residencia, tipo de escuela en que estudió, modalidad, edad del estudiante, rendimiento académico de cuarto y quinto año y trabajo. Por último, se realizó un plan de retención estudiantil para la URACCAN.

Palabras clave: abandono escolar; métodos de supervivencia; modelo de retención; rendimiento académico. (lleva punto y coma para que lo lea la base de datos).

\section{Summary}

School dropout affects all universities worldwide. This research explains the characteristics of students who drop out of URACCAN University, Nueva Guinea Campus, $2013-2017$ cut. In addition, a student retention model is proposed for this university. This investigation was carried out with two samples. The first objective was developed with all the 347 students who enrolled in the first year of their career in 2013. The second and third objective, work with 137 retired students. The mixed method and a correlational, phenomenological scope were used and due to its temporal location it is a cross-sectional study. In this study, it was found that the variables that have

1 Doctor en Matemáticas Aplicadas. Profesor de la Universidad de las Regiones Autónomas de la Costa Caribe Nicaragüense-Recinto Universitario Nueva Guinea. Email: sesmoizoog@gmail.com ORCID: https://orcid.org/oooo-0002-7093-9692

2 Doctor en Educación e Intervención Social. Profesor de la Facultad Multidisciplinaria de Chontales de la Universidad Nacional Autónoma de Nicaragua-Managua. Email: tonyromerong@hotmail.com ORCID: https://orcid.org/oooo-0oo3-3910-6980

Recibido: 21/01/2020 Aprobado: 10/03/2020

Medina López, M., y Romero Díaz, T. (2020). Modelo de supervivencia para analizar el abandono escolar de la URACCAN-Nueva Guinea. Ciencia E Interculturalidad, 26(01), 18-35. https://doi.org/10.5377/rci.v26i01.9881 
statistical influence on student dropout are the subject that the student chooses, the number of subjects failed and the average obtained in URACCAN. In turn, the variables studied and which do not statistically influence retirement are: gender, marital status, and area ofresidence, type of high school in which students graduated, modality, age of the student, academic performance of fourth and fifth year and work. Finally, a student retention plan was developed for URACCAN University.

Keywords: School Dropout; survival methods; retention model; academic performance.

\section{Introducción}

El abandono escolar es un problema para las universidades y que abarca varios aspectos de la misma como son el deterioro de los índices de la eficiencia terminal, índices de calidad institucional y pérdida de egresos por falta de pagos de aranceles. Según el Consejo Nacional de Universidades (CNU) en su informe de Rendición Social de Cuentas (2015 y 2016).

Tabla 1: Deserción de las universidades que pertenecen al CNU en el año 2015

\begin{tabular}{|l|l|l|l|l|l|}
\hline \multicolumn{1}{|c|}{ Universidad } & Matrícula Inicial & Matrícula Final & \% De Retención & \multicolumn{1}{|c|}{$\begin{array}{c}\text { Estudiantes } \\
\text { Retirados }\end{array}$} & \% Abandono \\
\hline AÑO 2015 & 111,207 & 94,946 & 85.38 & 16,261 & 14.62 \\
\hline AÑO 2014 & 109,352 & 94,322 & 86.26 & 15,030 & 13.74 \\
\hline AÑO 2013 & 104,319 & 90,826 & 87.07 & 13,493 & 12.93 \\
\hline AÑO 2012 & 103,206 & 91,705 & 88.86 & 11,501 & 11.14 \\
\hline AÑO 2011 & 99,063 & 88,113 & 88.95 & 10,950 & 11.05 \\
\hline PROMEDIO & 105,429 & 91,982 & 87.25 & 13,447 & 12.75 \\
\hline
\end{tabular}

Tabla modificada por el investigador a partir de la tabla obtenida en el informe de Rendición Social de Cuentas 2015.

El único estudio realizado en el recinto de Nueva Guinea es el realizado por López y Rojas (2010) en la carrera de Zootecnia, este estudio encontró que el porcentaje de deserción para esta carrera era del 40\%. Este estudio es importante porque permitió identificar las variables que más afectan a la deserción. Además, como el estudio fue realizado en varias carreras, permitió realizar comparaciones entre estos grupos. De igual manera, se hizo un seguimiento de estos grupos desde su matrícula hasta cinco años después, lo que permitió una mejor comprensión del fenómeno.

El resultado más importante de este estudio es que se encontró que las variables que tienen influencia estadística sobre el abandono escolar son: la carrera que el estudiante escoge, el número de asignatura reprobadas y el promedio obtenido en la URACCAN. A su vez las variables estudiadas y que no influyen estadísticamente en el retiro son: género, estado civil, zona de residencia, tipo de escuela en que estudió, modalidad, 
edad del estudiante, rendimiento académico de cuarto y quinto año y trabajo. Por último, se realizó un plan de retención estudiantil para la universidad URACCAN. Por último, es importante mencionar, que esta en una investigación concluida.

\section{Revisión de la literatura}

Torres (2007), plantea que los impactos sociales, institucionales y personales de la deserción pueden ser: elevación de los costos de formación, disminución de la calidad del proceso formativo, menor eficiencia de la carrera, crecimiento del desempleo y el no cumplimiento de la misión institucional (Citado en González, García, Izquierdo, y Torres, 2010).

De igual modo Patiño y Cardona (2012) expresan: "La deserción es un fenómeno complejo y de múltiples impactos negativos, tanto para el estudiante como para la propia universidad y, por extensión, para la región y la sociedad" (p. 2).

En Nicaragua hay varios estudios que analizan el fenómeno del abandono estudiantil en las universidades del país; sin embargo, en ninguno de ellos se hace uso de análisis estadístico.

Entre estos estudios se encuentran los siguientes: Aburto (2013) en UNANManagua FAREM Carazo, Fajardo (2013) en la Universidad Autónoma de Chinandega (UACH) y el realizado por Gutiérrez, Rosales y Espinoza (2015) en la UNAN-Managua. Estos estudios concluyen que las causas del abandono escolar se pueden agrupar en: aspectos académicos, económicos, motivacionales, normativos, familiares y laborales. Entre ellos están, movilidad por trabajo, choque de horario laboral y académico, bajos ingresos, falta de apoyo económico de la familia, poca orientación vocacional, desánimo y problemas familiares.

De acuerdo a los informes anuales que realizó el CNU (2012), (2013), (2014) y (2016), la deserción en las 10 universidades del CNU es el siguiente:

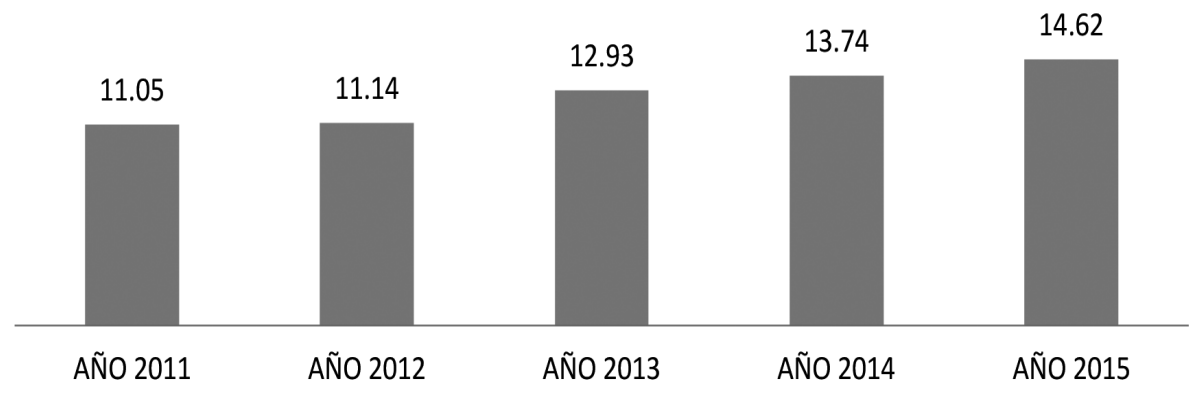

Figura 1: Retiro de los estudiantes en universidades del CNU. 
Como se puede ver el abandono universitario ha ido aumentando año con año, desde un 11.05 en el 2011 hasta parar en un 14.62 en el 2015.

En cuanto a los estudios realizados en la URACCAN se pueden mencionar: Jarquín, Sagastume y Argüello (2009), López y Rojas (2010), Castillo y Lee (2011). En estos estudios se menciona que las causas del abandono pueden ser socioeconómico, del propio sistema Universitario, de orden académico, personales y vocacionales.

Al representar la deserción en la URACCAN entre el 2011 al 2015 se obtiene el siguiente figura :

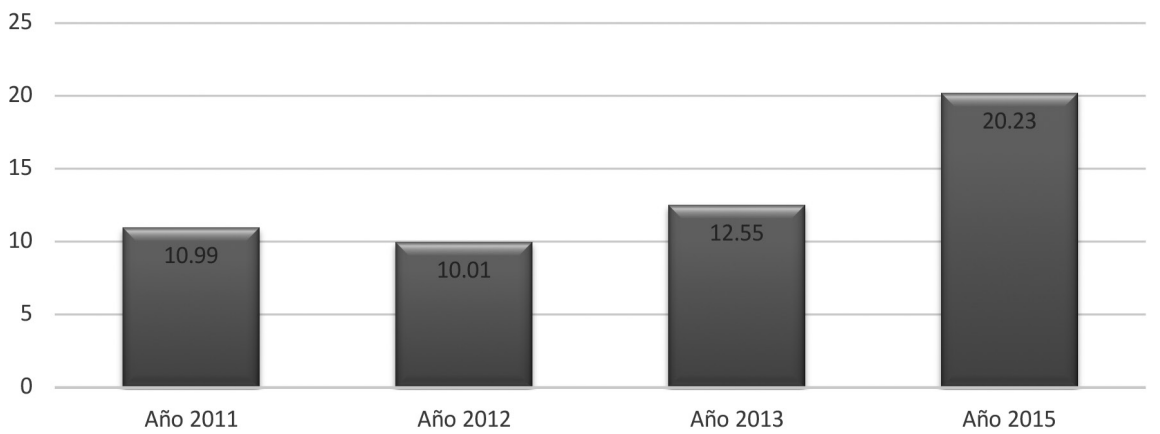

Figura 2: Abandono estudiantil de la URACCAN en los años 2011, 2012, 2013 y 2015

Aquí se puede ver que la deserción en la URACCAN ha venido aumentando a lo largo de los años representados con excepción del 2012.

Los métodos de supervivencia usados en este estudio fueron: tablas de mortalidad, procedimiento Kaplan-Meier y la Regresión de Cox.

Las Tablas de mortalidad de acuerdo con IBM SPSS Statistics (2018), consisten en subdividir el período de observación en intervalos de tiempo más pequeños en los cuales se observan a todos los sujetos del estudio, con la información obtenida se calcula la probabilidad de que un evento terminal tenga lugar dentro de ese intervalo.

El procedimiento Kaplan-Meier el cual, según Pérez (2001):

El procedimiento de Kaplan-Meier es un método de estimación de modelos hasta el evento en presencia de casos censurados. El modelo de Kaplan-Meier se basa en la estimación de las probabilidades condicionales en cada punto temporal cuando tiene lugar un evento y en tomar el límite del producto de esas probabilidades para estimar la tasa de supervivencia en cada punto temporal. 
En cuanto a la regresión de Cox se puede decir que, este modelo trabaja primordialmente con la función de riesgo (hazard function) y es utilizado para detectar relaciones existentes entre el riesgo que se produce en un determinado individuo en el estudio y algunas variables independientes y/o explicativas, por lo que este modelo nos permite evaluar dentro de un conjunto de variables cuáles tienen relación, influencia...sobre la función de riesgo y por ello también en la función de supervivencia, ya que ambas funciones están conectadas (Velasco y Muñoz, 2015).

\section{Materiales y método}

Esta es una investigación mixta debido a que hace uso de análisis y técnicas de recolección tanto cualitativas como cuantitativas. De acuerdo al alcance cuantitativo es un estudio correlacional. Pero de acuerdo al alcance cualitativo es un estudio fenomenológico. Otra parte importante a mencionar es que el estudio por su ubicación en el tiempo se clasifica como una investigación transversal debido a que se realizó en un solo momento.

La población de este estudio está constituida por los estudiantes que se matricularon en el primer año de su carrera en el 2013 en el recinto de la URACCAN Nueva Guinea (347), también Secretaria Académica y Bienestar Estudiantil de este recinto. La muestra fue dividida en dos partes. La primera parte está constituida por toda la población, los 347 estudiantes; la segunda parte la constituyen 137 estudiantes de los 214 que se retiraron de la población en estudio. De igual modo también están la Secretaría Académica y la responsable de Bienestar Estudiantil.

La recolección de los datos se hizo mediante el levantamiento de la información que los estudiantes matriculados en el primer semestre del 2013 poseían en registro académicos. Entre estos está la hoja de matrícula, los certificados de notas de cuarto y quinto año, los certificados de notas de los semestres cursados en la URACCANNueva Guinea. Por último, se realizó una entrevista a la secretaria académica y a la responsable de Bienestar Estudiantil.

En este estudio se hizo uso de los métodos estadísticos llamados métodos de supervivencia, los cuales son las tablas de mortalidad, método Kaplan-Meier y la regresión de Cox. También se hizo uso de un análisis descriptivo de las variables como son gráficos de barras e índices de tendencia central. Por último, se hizo uso del análisis cualitativo del fenómeno mediante las entrevistas realizadas. El análisis de resultados se realizó mediante los siguientes softwares: Excel, Word y SPSS.

La confiabilidad de la información recogida se llevó a cabo mediante el criterio de expertos obteniéndose un Alfa de Cronbach iguala a: por lo que se puede decir que posee una confiabilidad excelente. Por último, la variable dependiente de este estudio es la deserción y las independientes son: género, estado civil, zona de residencia, Tipo 
de escuela en la que estudió, modalidad, edad de los estudiantes, rendimiento académico de cuarto y quinto año, número de asignaturas reprobadas en la URACCAN, Promedio de nota obtenido en la URACCAN y trabajo.

\section{Resultados y discusión}

\section{Características generales que poseen los estudiantes que se matriculan en cada semestre de las carreras de la URACCAN-Nueva Guinea}

De los 347 estudiantes matriculados en el primer semestre de las carreras en estudio, el $61.7 \%$ de estos estudiantes abandonaron su carrera antes del primer semestre del año 2017. Este porcentaje representa un total de 214 estudiantes. Mientras que los que permanecieron representan un 38.3\%, lo que corresponde a 133 estudiantes.

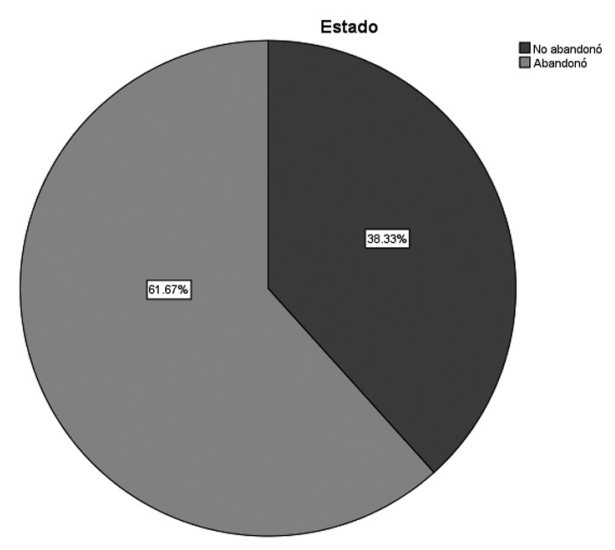

Figura 3: Abandono escolar en las carreras en estudio

Tabla 2: Abandono con respecto al sexo

\begin{tabular}{|c|c|c|c|c|c|c|}
\hline \multirow{2}{*}{ Sexo } & \multicolumn{4}{|c|}{ Estado } & \multirow{2}{*}{ Total } & \multirow[b]{2}{*}{$\%$} \\
\hline & No abandonó & $\%$ & Abandonó & $\%$ & & \\
\hline $\mathrm{F}$ & 76 & 57.14 & 109 & 50.93 & 185 & 53.31 \\
\hline M & 57 & 42.86 & 105 & 49.07 & 162 & 46.69 \\
\hline Total & 133 & 100 & 214 & 100 & 347 & 100.00 \\
\hline
\end{tabular}

De los 133 que no abandonaron sus carreras 76 de ellos eran varones (57.14\%) y 57 eran mujeres (42.86\%). De los 214 que abandonaron, 109 (50.93\%) eran mujeres; mientras que 105 eran varones (49.07\%). Es poca la diferencia por sexo entre los porcentajes de retirados y los que no lo hicieron. 


\section{EDUCACIÓN}

De los 249 que vienen del casco urbano, el 62.65\% (156) abandonaron su carrera. Además de los 98 estudiantes que son de fuera de la zona urbana el 59.18\% (58), abandonaron su carrera. Hay un mayor porcentaje de abandono de los estudiantes que vienen de la zona urbana.

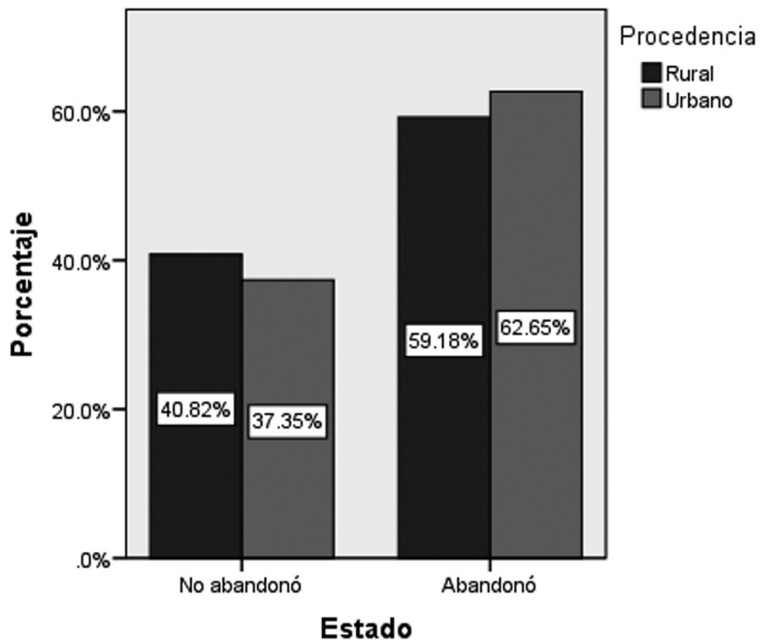

Figura 4: Procedencia contra el abandono escolar.

La carrera con más estudiantes fue la de administración ya que cuando esta carrera inició estaban divididos en dos secciones. Este grupo contenía 93 estudiantes en total, de los cuales, 56 abandonaron sus estudios, (26.17\%). El grupo con menos estudiantes fue el grupo de inglés con un total de 23 estudiantes, de estos solamente 12 se salieron de la carrera (5.61\%).

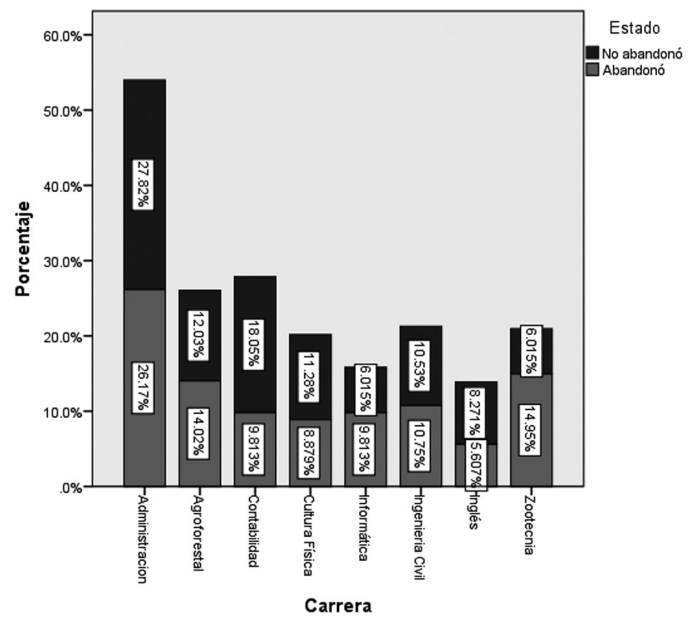

Figura 5: Abandono escolar distribuido por la carrera de estudio 


\section{Factores en el abandono estudiantil matriculados en el 2013 en la URACCAN- Nueva Guinea}

Esta etapa del estudio se llevó a cavo con la muestra de 137 estudiantes del total de 214 que abandonaron sus carreras.

Hay una predominancia de solteros con un total de 106 (77.37\%) sujetos. Luego, los que se encuentran en unión libre y casados tienen porcentajes semejantes con 10.95 y 11.68 para cada uno respectivamente, lo que representa 15 y 16 sujetos para ambos.

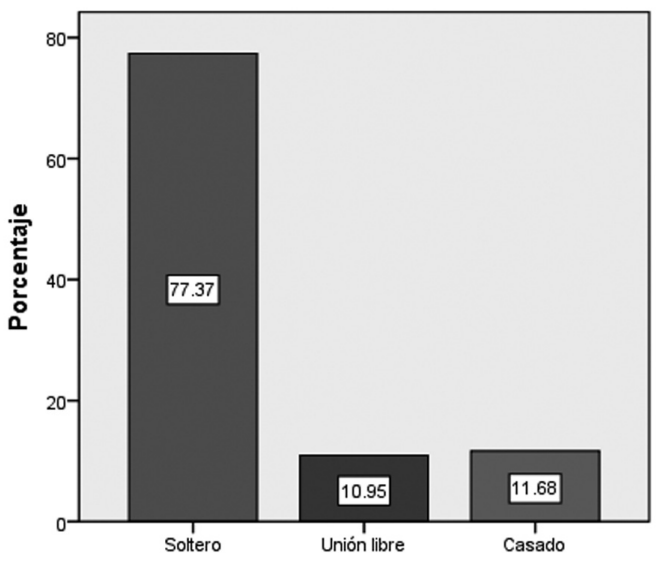

Figura 6: Estado civil de los retirados

$\mathrm{Al}$ analizar la edad de los estudiantes retirados se obtuvieron los siguientes resultados:

Tabla 3: Principales valores estadísticos de la edad

\begin{tabular}{|l|l|l|l|l|l|}
\hline & \multicolumn{1}{|c|}{ N } & \multicolumn{1}{|c|}{ Mínimo } & \multicolumn{1}{c|}{ Máximo } & Media & Desv. típ. \\
\hline Edad3 & 137 & 15.00 & 32.00 & 20.7518 & 3.57644 \\
\hline $\begin{array}{l}\text { N válido (según } \\
\text { lista) }\end{array}$ & 137 & & & & \\
\hline
\end{tabular}

La edad mínima es de 15 años para los retirados, mientras que la edad máxima es de 32 años. La media es de 20.75 años y la desviación estándar es de 3.58. El intervalo de edad con un nivel de confianza del $95 \%$ se encuentra en 20 y 22 años por lo que se concluye que los retirados son principalmente jóvenes. 


\section{EDUCACIÓN}

Del total de retirados el 56.20\% de los mismos estaban trabajando durante el período de estudio, lo cual corresponde a 77 individuos.

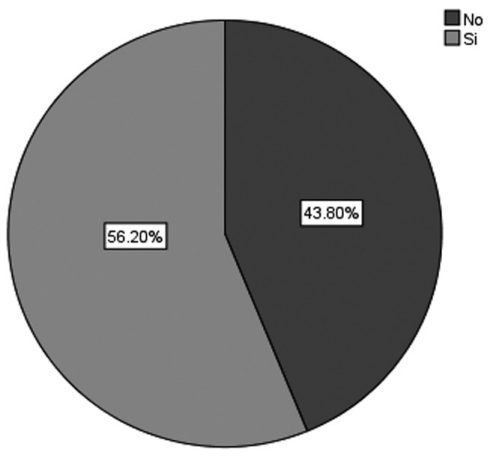

Figura 7: Trabajo de los estudiantes que abandonaron sus carreras

Al verificar la modalidad en la que estudiaron los estudiantes que se retiraron de su carrera se obtuvieron los siguientes resultados.

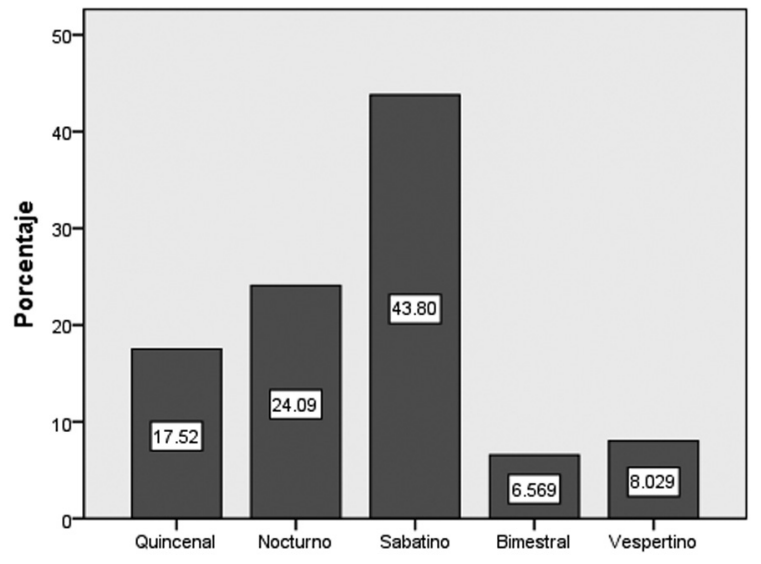

Figura 8: Modalidad de estudio

De esta figura se puede ver que la modalidad que más sujeto aportó a la muestra fue la sabatina, con un $43.8 \%$ lo que corresponde a 60 individuos. En segundo lugar, se tiene la modalidad nocturna, la cual tenía un porcentaje de $24.09 \%$, que es igual a 33 estudiantes.

Se encontraron individuos que no dejaron asignaturas, y otros que reprobaron 11 en total. El promedio de asignaturas reprobadas fue de 2.8 y la desviación estándar es de 2.8, con lo cual queda un intervalo de confianza de 2.33 a 3.27. Este resultado muestra que el porcentaje de asignaturas que reprueba el desertor es bajo. 
Se encontraron individuos que no registraron ninguna nota, los cuales tenían como promedio cero. El promedio más alto de nota registrada es 88.52. Al mismo tiempo, la media es de 44.41. Este promedio bajo es debido a los individuos con promedio cero.

Tabla 4: Promedio de nota de la URACCAN

\begin{tabular}{llllll}
\hline & $\mathrm{N}$ & Mínimo & Máximo & Media & Desv. típ. \\
\hline Promedio de URACCAN & 137 & .00 & $\mathrm{~V}$ & 44.4144 & $33.24063^{`}$ \\
N válido (según lista) & 137 & & & & \\
\hline
\end{tabular}

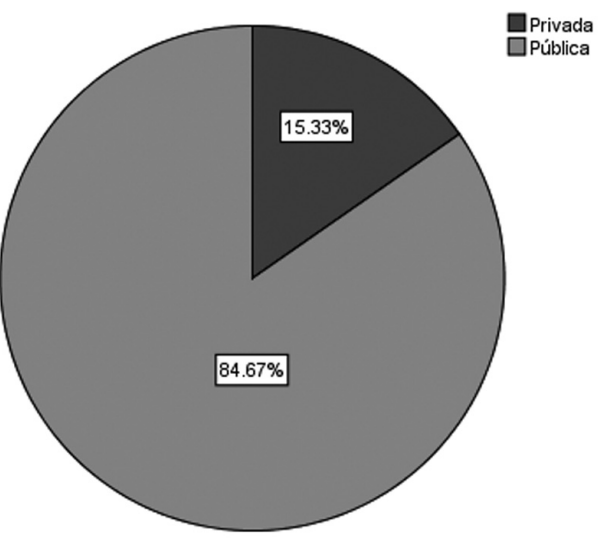

Figura 9: Escuela pública o privada

El $84.67 \%$ de los retirados pertenecen a las escuelas públicas (116). Mientras que los que estudiaron en las escuelas privadas constituyen $15.33 \%$, lo cual es igual a 21 estudiantes. Esto se debe a que la mayor parte de los estudiantes del municipio son de escuelas públicas.

La nota mínima para el cuarto y el quinto año son 52.8 y 58.82 respectivamente. Estas notas, aunque están reprobadas, los estudiantes aprobaron estas asignaturas mediante el examen de reparación. Los promedios máximos son de 94.2 y 94.5 respectivamente. Estos promedios son bastante altos, lo que demuestra que entre los retirados hay estudiantes con notas excelentes. La media para estas variables fue de 74.93 y 76.98 , la cual no es tan baja.

\section{Análisis de supervivencias en el abandono escolar}

Esta tabla de mortalidad se realizó con toda la población (347). En ella se ve que la mayor cantidad de eventos terminales ocurre dentro del primer período. De los 347 matriculados 127 (37\%) se retiraron durante el primer periodo. Así mismo, la probabilidad que un estudiante no deserte durante el primer año es de 60\%, mientras que de que termine su carrera es de $38 \%$. 


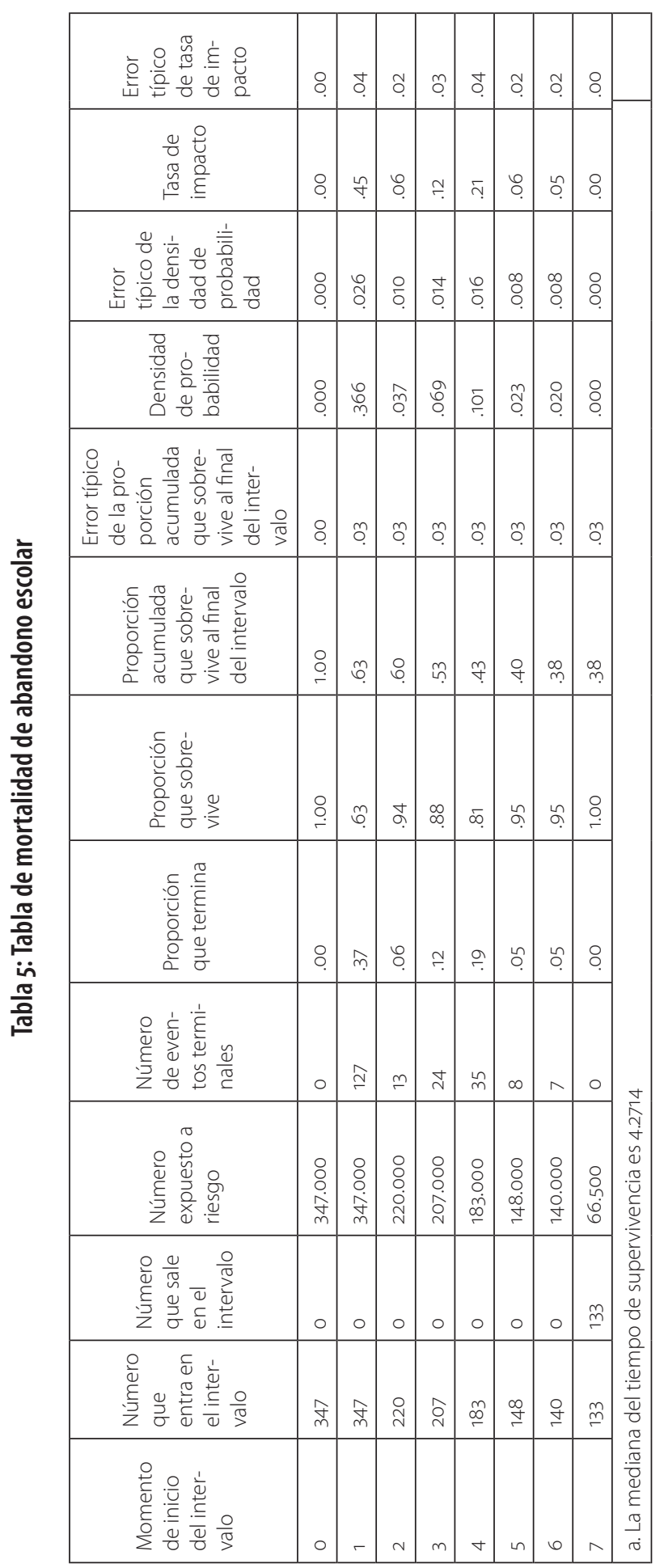




\section{Análisis de supervivencia mediante el método Kaplan Meyer}

Las carreras con menor abandono la conforman: Administración, Contabilidad, Cultura Física, Ingeniería Civil e Inglés. Mientras que las que poseen mayor abandono son Agroforestal, Informática y Zootecnia.

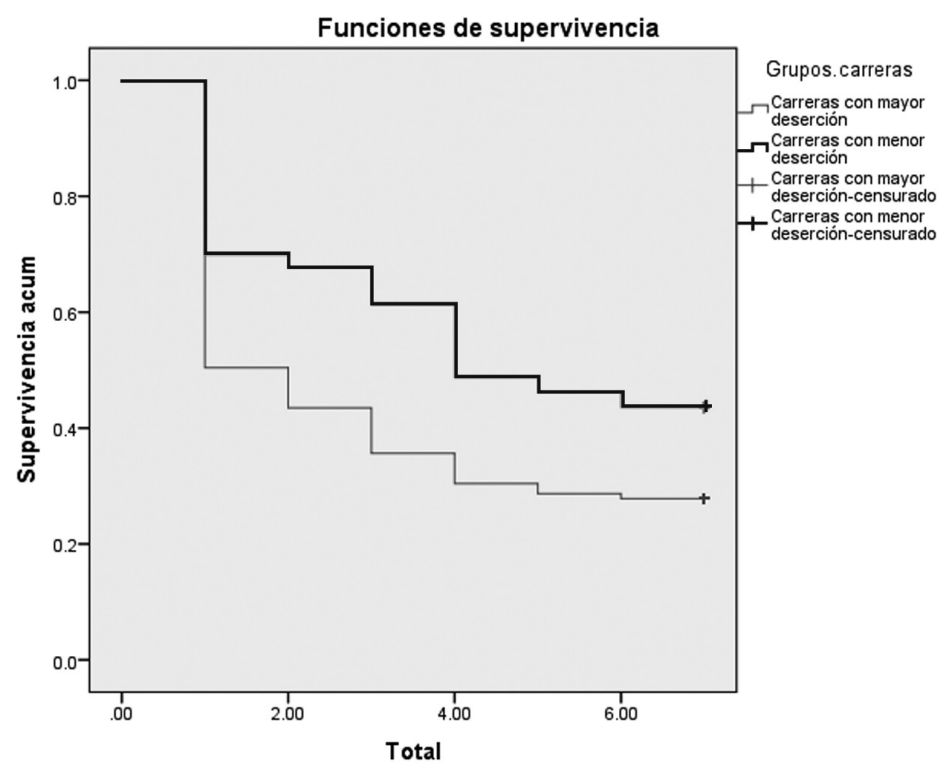

Figura 10: Función de supervivencia por grupos de carreras.

Hay una clara diferencia entre las carreras con un mayor abandono y las que poseen mejor retención. Al calcular el nivel de significancia se ve que este valor da como resultado un valor inferior a 0.05 por lo tanto se concluye que ambos grupos son diferentes entre sí.

Las gráficas que representan a estudiantes que vienen de fuera del casco urbano y los que vienen de dentro son similares entre sí. Sin embargo, el abandono escolar es mayor en las personas que vienen de adentro. 


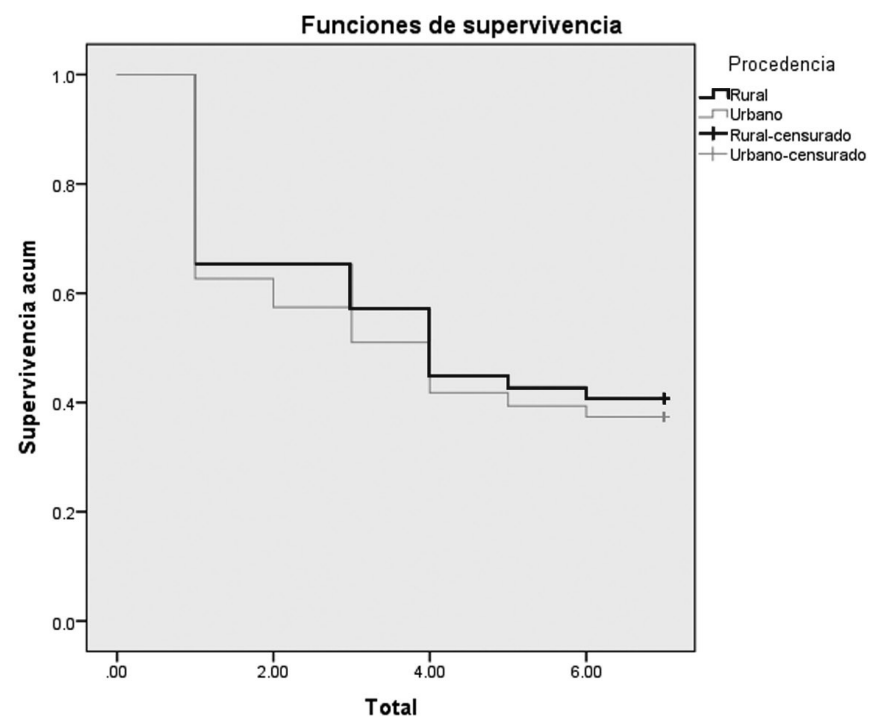

Figura 11: Función de supervivencia por procedencia de los estudiantes

Como el nivel de significancia en la prueba estadística da por encima de 0.05 no hay diferencia significativa entre estos grupos.

La modalidad con menor deserción es la bimestral mientras que la modalidad con mayor deserción es la quincenal.

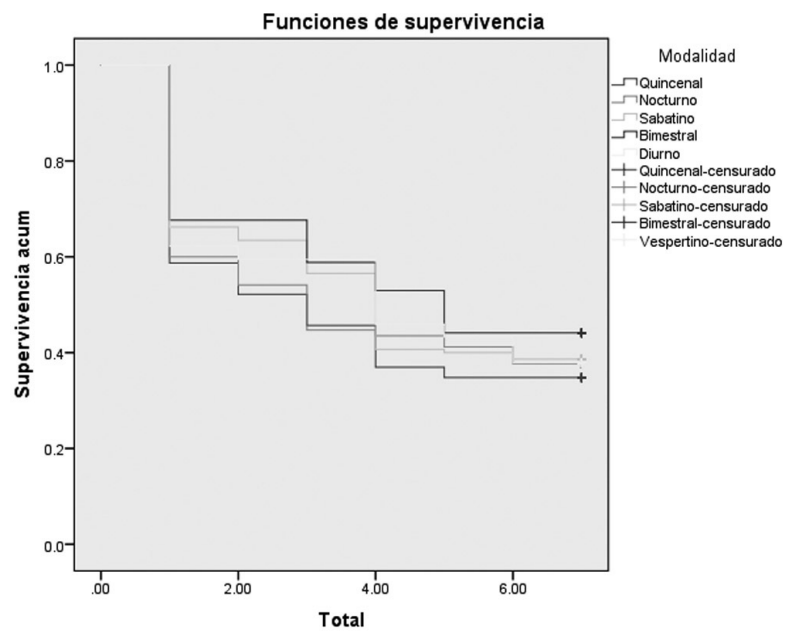

Figura 12: Función de supervivencia separada por modalidad 
$\mathrm{Al}$ analizar el valor de significancia para esta variable se obtienen un valor mayor a 0.05 por lo que se concluye que no hay influencia de esta variable sobre la deserción.

\section{Análisis de supervivencia del abandono escolar mediante el método de Regresión de Cox}

Se utilizó el método de regresión logística hacia adelante.

En la tabla siguiente se puede ver que el valor de significancia en menor a 0.05 lo que indica que al menos una variable influye en el abandono estudiantil.

Tabla 6: Pruebas omnibus sobre los coeficientes del modelob

\begin{tabular}{|c|c|c|c|c|c|c|c|c|c|c|}
\hline \multirow[t]{2}{*}{ Paso } & \multirow{2}{*}{$\begin{array}{l}-2 \text { log de } \\
\text { la verosi- } \\
\text { militud }\end{array}$} & \multicolumn{3}{|c|}{ Global (puntuación) } & \multicolumn{3}{|c|}{$\begin{array}{l}\text { Cambio desde el paso } \\
\text { anterior }\end{array}$} & \multicolumn{3}{|c|}{$\begin{array}{l}\text { Cambio desde el bloque } \\
\text { anterior }\end{array}$} \\
\hline & & $\begin{array}{l}\text { Chi- } \\
\text { cuadrado }\end{array}$ & $\mathrm{gl}$ & Sig. & $\begin{array}{l}\text { Chi- } \\
\text { cuadrado }\end{array}$ & $g l$ & Sig. & $\begin{array}{l}\text { Chi- } \\
\text { cuadrado }\end{array}$ & $g l$ & Sig. \\
\hline $1 a$ & 2386.817 & 9.400 & 1 & .002 & 8.919 & 1 & .003 & 8.919 & 1 & .003 \\
\hline
\end{tabular}

En el primer paso se agregó la variable carrera y ninguna otra variable.

Tabla 7: Variables en la ecuación

\begin{tabular}{llllllllll|}
\hline & & B & ET & Wald & gl & Sig. & \multirow{2}{*}{$\begin{array}{l}\text { Exp(B) } \\
\text { Inferior }\end{array}$} & \multicolumn{2}{l}{$95.0 \%$ IC para Exp(B) } \\
\cline { 8 - 10 } & & & & & & Superior & \\
\hline Paso 1 & $\begin{array}{l}\text { Grupos. } \\
\text { carreras }\end{array}$ & .430 & .141 & 9.261 & 1 & .002 & 1.537 & 1.165 & 2.027 \\
\hline
\end{tabular}

Luego de este paso quedaron las variables sexo y procedencia fuera de la ecuación. Otro valor importante a tomar en cuente en la tabla anterior es el valor Exp(B) el cual significa que un estudiante que pertenece a las carreras de Zootecnia, Informática y Agroforestal tiene $53.75 \%$ más probabilidad de abandonar sus carreras que los otros.

También se aplicó la regresión de Cox a otras variables, pero con una muestra menor ya que no fue posible conseguir la información de todos los estudiantes que participan en el estudio. Las variables que se analizarán son: promedio académico del estudiante en la URACCAN, número de clases reprobadas en la URACCAN, estado civil del estudiante, edad del estudiante por intervalos, modalidad en la que estudió la secundaria, promedio del quinto año, promedio del cuarto año, escuela pública o privada, trabajo, edad en forma numérica. 
Hay influencia de al menos una variable en el abandono escolar ya que los valores de significancia son menores a $0.05 \%$.

Tabla 8: Pruebas omnibus sobre los coeficientes del modeloc

\begin{tabular}{|c|c|c|c|c|c|c|c|c|c|c|}
\hline \multirow[t]{2}{*}{ Paso } & \multirow{2}{*}{$\begin{array}{l}-2 \text { log de } \\
\text { la verosi- } \\
\text { militud }\end{array}$} & \multicolumn{3}{|c|}{ Global (puntuación) } & \multicolumn{3}{|c|}{$\begin{array}{l}\text { Cambio desde el paso } \\
\text { anterior }\end{array}$} & \multicolumn{3}{|c|}{$\begin{array}{l}\text { Cambio desde el blo- } \\
\text { que anterior }\end{array}$} \\
\hline & & $\begin{array}{l}\text { Chi- } \\
\text { cuadrado }\end{array}$ & $\mathrm{gl}$ & Sig. & $\begin{array}{l}\text { Chi- } \\
\text { cuadrado }\end{array}$ & gl & Sig. & $\begin{array}{l}\text { Chi- } \\
\text { cuadrado }\end{array}$ & $\mathrm{gl}$ & Sig. \\
\hline $1 a$ & 1134.493 & 18.297 & 1 & .000 & 17.589 & 1 & .000 & 17.589 & 1 & .000 \\
\hline $2 b$ & 1129.612 & 22.665 & 2 & .000 & 4.881 & 1 & .027 & 22.470 & 2 & .000 \\
\hline \multicolumn{11}{|c|}{ a. Variables introducidas en el paso número 1: Promedio-ura. } \\
\hline \multicolumn{11}{|c|}{ b. Variables introducidas en el paso número 2: Clases-reprobadas. } \\
\hline \multicolumn{11}{|c|}{ c. Bloque inicial número 1. Método = Por pasos hacia adelante (Wald). } \\
\hline
\end{tabular}

Las variables que están al inicio en la ecuación son el promedio escolar de los estudiantes en la URACCAN, y la otra es el número de materias reprobadas.

Tabla 9: Variables en la ecuación

\begin{tabular}{|c|c|c|c|c|c|c|c|c|c|}
\hline & & \multirow[t]{2}{*}{$B$} & \multirow[t]{2}{*}{ ET } & \multirow[t]{2}{*}{ Wald } & \multirow[t]{2}{*}{$\mathrm{gl}$} & \multirow[t]{2}{*}{ Sig. } & \multirow{2}{*}{$\begin{array}{l}\text { Exp(B) } \\
\text { Inferior }\end{array}$} & \multicolumn{2}{|c|}{ 95.0\% IC para $\operatorname{Exp}(B)$} \\
\hline & & & & & & & & Super & \\
\hline \multirow[t]{2}{*}{ Paso 1} & Promedio.ura & -.014 & .003 & 17.214 & 1 & .000 & .987 & .980 & .993 \\
\hline & Promedio.ura & -.027 & .008 & 12.633 & 1 & .000 & .973 & .959 & .988 \\
\hline Paso 2 & $\begin{array}{l}\text { Clases.repro- } \\
\text { badas }\end{array}$ & -.172 & .087 & 3.875 & 1 & .049 & .842 & .710 & .999 \\
\hline
\end{tabular}

De lo anterior se concluye que las únicas variables que influyen en el abandono escolar son: el promedio académico de las notas de la URACCAN, el número de clases reprobadas en el recinto y la carrera elegida, la cual fue analizada anteriormente.

\section{Retención estudiantil en estudiantes de la URACCAN-Recinto Universitario Nueva Guinea}

De la entrevista se obtuvo que las razones por las que los estudiantes abandonan son: falta de vocación, problemas económicos tantos de los estudiantes como de sus familias, por el hecho de conseguir empleo, por falta de empleos ya que no pueden costear sus estudios, por falta de motivación durante el primer año.

La universidad URACCAN no cuenta con un plan de retención estudiantil, sin embargo, en algunas de sus políticas aparecen acciones para combatir el abandono escolar tales como: Dar seguimiento personalizado a cada uno de los estudiantes, se han creado planes extracurriculares de retención estudiantil, la academia ha creado 
planes de motivación, se han iniciado programas de cultura y deportes, reuniones con estudiantes becados, se han realizado tres asambleas con estudiantes becados $\mathrm{y}$ círculos de estudio grupales guiados por estudiantes monitores con el apoyo de los docentes de cada asignatura.

\section{Conclusiones}

Las principales características que poseen los matriculados en 2013 son: El 61.7\% abandonaron sus estudios, el $71.76 \%$ era del casco urbano de Nueva Guinea, no hay relación entre la procedencia y el abandono escolar, la carrera con mayor porcentaje de abandono fue la Administración de Empresas con un 26.17\% y la carrera con menor porcentaje de retención es Zootecnia, con un $20 \%$.

Factores que influyen en el abandono escolar: $\mathrm{El} 58.4 \%$ de los retirados lo hicieron durante el primer periodo de evaluación, el 6o.6\% eran mujeres, el $77.37 \%$ son solteros, el $69.35 \%$ posee edad entre 15 años y 22 años, el $56.20 \%$ poseían trabajo, el $75.18 \%$ eran del casco urbano, todos los estudiantes de las carreras Zootecnia y Contabilidad eran del casco urbano, el $43.80 \%$ pertenecen a la modalidad sabatina, el promedio de asignaturas reprobadas anda entre 2.33 y 3.27 , el promedio de notas en la URACCAN están reprobadas con un $44.41 \%$, el $84.67 \%$ estudiaron en escuela pública y por último, el promedio del cuarto y del quinto año se encuentran entre 74.93 y 76.98 por ciento respectivamente, lo que constituye una nota aprobada.

De los métodos de supervivencia utilizados se concluye que: La probabilidad de que un estudiante se retire durante el primer año es de $37 \%$ y de que termine su carrera es de $38 \%$, la probabilidad de que se retire en su último año es de un $5 \%$, las carreras con menor abandono escolar son: Administración, Contabilidad, Cultura Física, Ingeniería Civil e Inglés, las carreras con mayor abandono escolar son: Agroforestal, Informática y Zootecnia, las variables que influyen en el abandono escolar son: el promedio de nota obtenido en la URACCAN y el número de clases reprobadas y la carrera elegida, de igual forma, las que no influyen en el abandono escolar son: sexo, procedencia, y modalidad, estado civil, edad, promedio de cuarto y quinto año, trabajo, tipo de escuela (pública o privada) y modalidad en que estudió la secundaria.

Con respecto al plan de retención: La URACCAN no cuenta con un plan de retención estudiantil, entre las causas de abandono están: falta de educación vocacional, falta de empleo y falta de motivación, la universidad realiza acciones encaminadas a disminuir el abandono mediante el seguimiento, monitoreo de las carreras y programas culturales y deportivos. 


\section{EDUCACIÓN}

\section{Agradecimiento}

Esta publicación obtuvo el financiamiento de: El Fondo de Asistencia Internacional de los Estudiantes y Académicos Noruegos (SAIH).

\section{Lista de referencia}

Aburto, P. (2013). Estudio de la deserción en la Facultad Regional Multidisciplinaria (FAREM-Carazo). Proyecto ALFA GUÍA, 12-25.

Castillo, L., y Lee, B. (2011). La eficiencia terminal educativa en URACCAN recinto Las Minas, generaciones 1995-2002. Ciencia e Interculturalidad, 8(1), 25-40.

CNU (2012). Informe Rendición Social de Cuentas año 2011. Managua.

CNU (2013). Informe de Rendición Social de Cuentas año 2012. Managua.

CNU (2014). Rendición Social de Cuentas. Managua.

CNU (2016). Rendión Social de Cuentas 2015. Managua.

Fajardo, A. (2013). Factores que tienen presencia en a deserción estudiantil en los alumnos de primer ingreso de la Universidad Autónoma de Chinandega (UACH) en el período 2010 - 2011. Proyecto ALFA GUÍA, 24-28.

González Rodríguez, J. A., García Gómez, T., Izquierdo Dorta, C., y Torres García, A. (2010). Deserción estudiantil ¿Los que abandonan, son ellos o nosotros? (Spanish). Pedagogía Universitaria, 15(2), 7.

Gutiérrez, M., Rosales, Y., y Espiñoza, M. (2015). Deserción Académica de los estudiantes de la carrera de Educación Comercial. (Tesis de licenciatura), UNAN-Managua: Nicaragua.

IBM SPSS Statistics. (20 de Septiembre de 2018). Ayuda-IBM SPSS Statistics. Obtenido de Ayuda - IBM SPSS Statistics: https://www.youtube.com

Jarquín, I., Sagastume, R., y Argüello, J. (2009). Factores de incidencia en el abandono estudiantil, URACCAN Las Minas. Ciencia e Interculturalidad, 5(2), 50-62.

López, A., y Rojas, N. (2010). Factores de abandono estudiantil en la carrera de Ingeniería en Zootecnia, recinto Nueva Guinea. Ciencia e Interculturalidad, 7(2), 114-124. 
Patiño Garzón, L., y Cardona Pérez, A. M. (2012). Revisión de algunos estudios sobre la deserción estudiantil universitaria en Colombia y Latinoamérica (Spanish). Theoría; Ciencia, Arte y Humanidades, 21(1), 9-20.

Pérez, C. (2001). Técnicas estadísticas con SPSS. Madrid: Prentice Hall.

Velasco, P., y Muñoz, J. (2015). Modelo de regresión de Cox y sus aplicaciones biosanitarias. Tesis de grado de Estadística, Universidad de Savilla: Sevilla. 